\title{
The impact of kidney failure on blood pressure
}

\section{Niamh Goulding}

Department of Physiology, UCC

\section{Introduction}

The aim of my research is to look at kidney failure and the problems associated with it, in particular high blood pressure (hypertension). The main organ of the excretory system is the kidney. The kidneys are paired organs lying in the posterior abdominal wall on either side of the vertebral column. They are covered in a tough fibrous capsule.

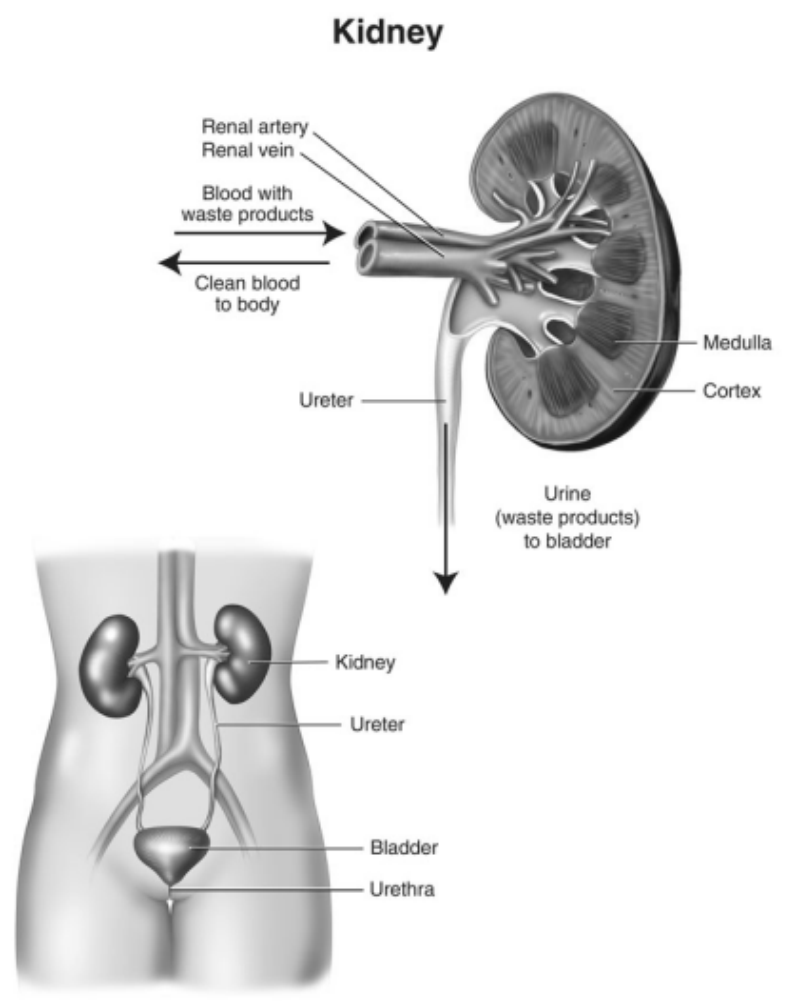

Figure 1: Kidney position and structure

The function of the kidneys is maintaining body fluid balance. They do this by filtering blood to produce urine, getting rid of waste material and keeping nutrients in the body. This particular function is carried out by structures called nephrons within the kidney. Each kidney contains approximately 1 million nephrons. 


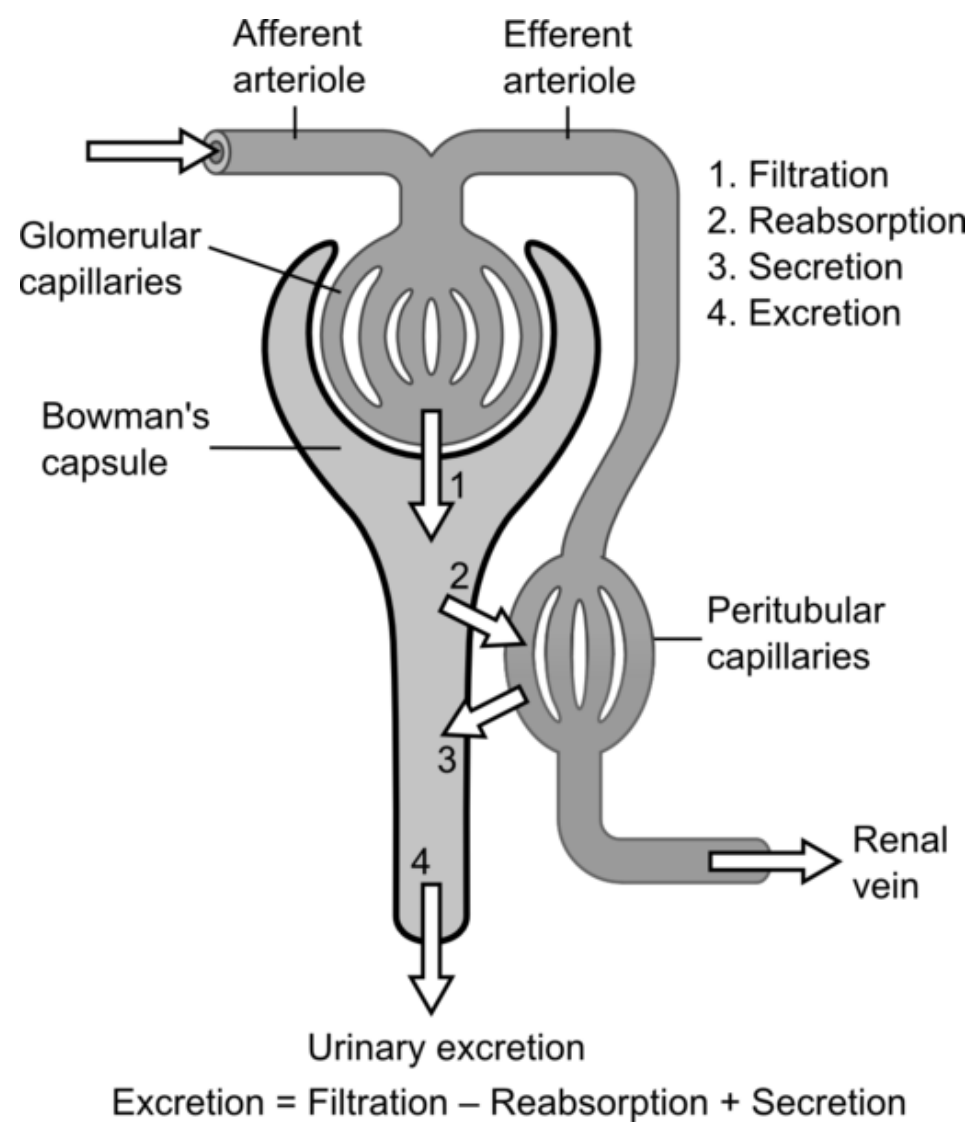

Figure 2: Nephron structure

\section{Urine Formation}

Urine is made in the nephron. Blood enters into the nephron through a small artery, the afferent arteriole, and from there into a highly permeable structure called the glomerulus.

The glomerulus rests on top of a sieve like structure called Bowman's capsule. Slits in Bowman's capsule allow small molecules (e.g., glucose, water, sodium) to pass through its wall and into the nephron. Larger substances (e.g., protein and red blood cells) cannot pass through the small slits and leave the glomerulus through the efferent arteriole. The smaller substances that are filtered enter into the tubules of the nephron. Here the fluid or filtrate can be altered in two ways:

$\square$ Reabsorption - a process by which something is retained in the body by moving it across the tubules and back into the blood

Secretion - this involves the movement of a substance from the blood into the tubules. At the end of all of these alterations what remains is urine to be excreted.

Probably the most important part of urine formation is what is called glomerular filtration, i.e., the filtration process that occurs across the glomerulus. In a clinical environment, in a case where a patient is suspected of having some sort of kidney impairment, a test called 


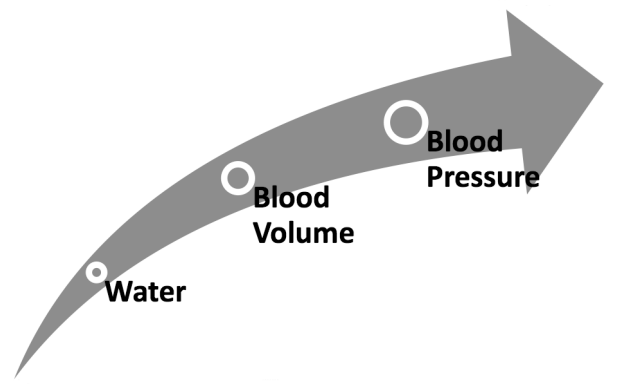

Figure 3: Relationship between body water and blood pressure

glomerular filtration rate or GFR is carried out. This test is an index of kidney function, which tells us how well the patient's kidneys are functioning. So, if a patient has renal failure (loss of kidney function) this will cause a very visible decrease in GFR.

\section{Body water and blood pressure are linked}

The main component of our blood is water. The amount of water in the body determines blood volume (i.e., the amount of circulating blood we have). If we drank $1 \mathrm{~L}$ of water over a 15 minute period, this water would make its way into our bloodstream. We effectively now have an extra litre of blood because of the presence of this extra fluid. Our blood volume has now increased and this has the direct effect of increasing our blood pressure, as our body tries to deal with the extra volume of blood.

So to combat the rise of blood pressure our kidneys react, via reflex neural and hormonal mechanisms, by excreting more water to decrease the amount in our blood. In this way the renal system has knock-on effects on the cardiovascular system. By controlling water loss (water secretion) and water retention (water reabsorption), the kidneys regulate blood pressure.

\section{Renal Nerves}

Our kidneys are innervated by a particular type of nerve called sympathetic nerves. When these nerves are stimulated (sympatho-excitation) the major effect is to constrict the afferent arteriole. In other words, stimulation of sympathetic nerves makes the arteriole entering the nephron smaller in diameter. This means less blood enters into the glomerulus and, as a direct result, a decrease in filtration occurs and less urine is produced. Conversely if sympathetic nerve stimulation is decreased, (sympatho-inhibition) less constriction is initiated. This results in dilation or widening of the arteriole into the glomerulus. This allows more blood flow into the glomerulus, more filtration occurs, and more urine is produced. 
Times when the body would want to inhibit these nerves (sympatho-inhibition) are times when we need to increase GFR, for example as described earlier when we drink a lot of water and we need to excrete it. So, we would want to increase stimulation of these nerves when we need to retain water, for example if we were dehydrated. If these relationships are disturbed, then there is a progression into a state of hypertension, leading to an increased risk of heart failure and stroke. An essential element in this feedback relationship is to understand whether damage to or disease of the kidney itself can act as an initiator of sympathetic drive, leading to a deranged cardiovascular homeostasis. Homeostasis as defined by the French physiologist Claude Bernard (1813-78), refers to the stability and maintenance of the internal environment ('milieu intérieur') of the body. Homeostasis involves physiological processes by which the internal systems of the body are maintained in equilibrium despite variations in the external conditions.

\section{Renal Receptors}

Within the kidney there are specialised sensory receptors (responsible for the pain associated with kidney stones). These sensors are called mechanoreceptors and chemoreceptors. The mechanoreceptors are sensitive to pressure changes within the kidney and information from here will be relayed to the brain. The chemoreceptors are chemical sensors that are particularly sensitive to salt $(\mathrm{NaCl})$. Again, information from these sensors is relayed to the brain. Depending on whether pressure is high or low, or whether dietary salt levels are high or low, the brain will respond by causing the release of particular hormones or by activating or inhibiting the sympathetic nerves.

\section{Renal Disease}

Chronic renal disease (CRD) is an insidious deterioration of kidney function which becomes apparent after the loss of some $90 \%$ of nephrons. Its occurrence is increasing at an alarming rate in the increasing population of diabetic type 2 and obese patients. CRD is associated with a hypertension resistant to management by conventional drugs.

When CRD gets to the stage where the kidneys function so poorly that they can no longer keep you alive, a kidney transplantation is sometimes the only option. This often involves the replacement of the failed kidney with a healthy donor kidney. However, removing the diseased kidney adds to the risks during surgery, so what often happens is the healthy donor kidney is simply grafted into place as can be seen in Figure 4.

In patients with this type of transplantation, where the diseased kidney is not removed, the drug resistant hypertension is most common. This led researchers to believe that the cause of the hypertension may be arising from the diseased kidney. In fact, the most prevalent theory is that the diseased kidney's sensory receptors are over-activated. Their over- 


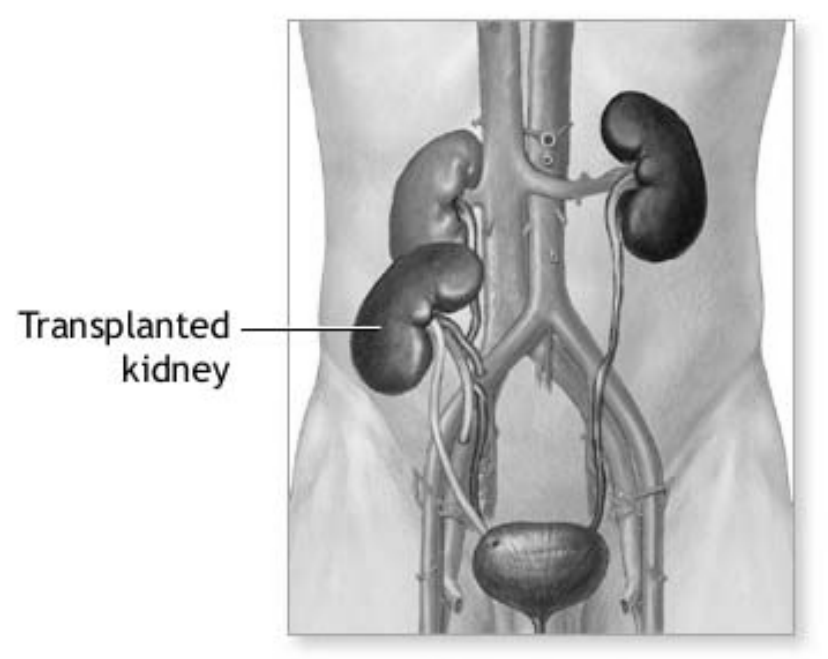

Figure 4: Kidney transplant

activation causes over-activation of the sympathetic nerves, which will have the knock-on effect of increasing blood pressure and leading to a hypertensive state.

\section{My Project}

My research project examines why such resistant hypertension exists with renal failure and possible treatments for it. There is a strong association between hypertension and chronic renal disease and, although the underlying mechanisms are unclear, experimental and clinical studies have implicated the sympathetic nervous system (the nerves innervating the kidney). There are sensory receptors within the kidney which, when challenged with physiological stimuli (e.g., increased body water or salt), can increase or decrease sympathetic nerve activity as part of the reno-renal reflex, as previously described.

\section{Research Background}

Previous studies in the rat have shown renal injury to elicit an immediate and sustained increase in blood pressure, plasma noradrenaline secretion (a direct marker of sympathetic nerve activity) and renal sympathetic nerve activity. Studies in human subjects, found that the rate of sympathetic nerve activity was three times higher in renal transplant patients who retained their diseased kidneys, compared to normal subjects. Moreover, if, during transplant surgery, the diseased kidney was removed, the rate of sympathetic activity could not be distinguished from the control subjects. The consensus is that this inappropriate and excessive stimulation of the sensory receptors within the kidney by the disease process elicits increased sympathetic drive, contributing to a state of hypertension. 


\section{My PhD research}

To examine the cardiovascular effects of CRD, metabolic tests were carried out on laboratory rats with a drug-induced renal injury. Metabolic tests involve housing the rat in a specially designed cage that allows me to take various measurements, e.g., water intake, urinary output. Analysis of urine samples and plasma samples allowed me to quantify the effect of CRD on the rats compared to normal healthy control rats. A 70\% decrease in individual kidney functions (i.e., GFR, sodium excretion rates, water excretion rates) was observed in the CRD rats.

Next, to examine whether CRD could have an effect on the sensory receptors of the kidney and the kidneys control of blood pressure, I looked at levels of a substance called noradrenaline. This is a substance that is released during sympathetic nerve activation. CRD rats had increased levels of noradrenaline in the blood (plasma). This suggested that over-activation of renal nerves was occurring, as more noradrenaline was being secreted.

The effects of CRD were further examined during surgical experiments. This type of experiment allowed me to directly measure renal nerve activity during different physiological challenges. During my experimental protocol, the rat's BP is increased and decreased to observe how the rat's cardiovascular system and renal system reacts. In the control rats (healthy rats) blood pressure was corrected quickly and the reno-renal reflex was intact. The CRD group's reflex regulation of blood pressure was blunted, however, and the reno-renal reflex was greatly inhibited. Sympathetic nerve activity was at a high level throughout the experiment and was neither activated further nor inhibited, as would be expected in healthy rats. These results indicate ineffective cardiovascular control of blood pressure. Such impairment could contribute, if only in part, to the hypertension associated with CRF.

To further analyse the source of the sympatho over-activation I carried out further metabolic tests, this time with prior renal denervation (loss of nerve supply). This in theory would block the sensory signals arising from the diseased kidney and, as a result, decrease sympathetic nerve activation and BP. In these denervated rats sympathetic activation was reduced suggesting that this is a very possible cause of irregular blood pressure control in CRD patients. 


\section{Conclusion}

My research thus far has had a number of milestone findings:

1. CRD has associated with it increased noradrenaline rates which could be a direct result of sympathetic nerve over-activation

2. CRD rats exhibit dysregulation of many cardiovascular and renal reflexes

3. Denervation in CRD rats appears to negate the effects of sympathetic over-activation.

The most important thing to be taken from my $\mathrm{PhD}$ research is that renal denervation is a viable treatment for drug resistant hypertension. This treatment can obliterate the irregular and incorrect sensory signals being sent from the diseased kidney and which cause hypertension. In fact in recent months my data has been translated into man, with renal denervation in humans with drug-resistant hypertension normalised blood pressure. In the coming months I will continue my experiments to further bolster my findings to date and also to investigate the sensory transduction mechanisms involved in drug- resistant hypertension. In doing so it may be possible to isolate a particular drug treatment for this hypertension.

Thanks to my supervisors Professor Edward J. Johns, Dr. John Mackrill and Dr. Therese Ruane-O'Hora, Dr. Chun Long Huang for his invaluable experience and my colleagues, Claire Thompson, Evelyn Flanagan and Maria Buckley. 